\title{
Phenylephrine to Treat Hypoxemia during One-Lung Ventilation in a Pediatric Patient
}

\author{
Brian Schloss ${ }^{1}$ David Martin ${ }^{1} \quad$ Allan Beebe $^{2} \quad$ Jan Klamar $^{2} \quad$ Joseph D. Tobias $^{1}$ \\ ${ }^{1}$ Department of Anesthesiology and Pain Medicine, Nationwide \\ Children's Hospital, Columbus, Ohio, United States \\ 2 Department of Orthopedic Surgery, Nationwide Children's Hospital, \\ Columbus, Ohio, United States

\begin{abstract}
Address for correspondence Brian Schloss, MD, Department of Anesthesiology and Pain Medicine, Nationwide Children's Hospital, 700 Children's Dr, Columbus, OH 43205, United States (e-mail: brian.schloss@nationwidechildrens.org).
\end{abstract}

Thorac Cardiovasc Surg Rep 2013;2:16-18.

\begin{abstract}
\section{Keywords}

- pediatric

- hypoxia

- one-lung ventilation

To improve surgical visualization and facilitate the procedure, one-lung ventilation (OLV) is frequently used during thoracic surgery. Although generally well tolerated, the ventilation-perfusion inequality induced by OLV may lead to a decrease in oxygenation and, at times, hypoxemia. Effective treatment algorithms and strategies are necessary for the treatment of hypoxemia during OLV to ensure that the technique can be continued without interruption and allow for completion of the surgical procedure. Treatment strategies may include applying positive end expiratory pressure to the nonoperative lung, continuous positive airway pressure or low flow oxygen insufflation to the operative lung, decreasing anesthetic agents that interfere with hypoxic pulmonary vasoconstriction (HPV), or switching to total intravenous anesthesia. Although less commonly employed, $\alpha$-adrenergic agonists may also improve oxygenation during OLV by augmenting HPV. We present a 12-year-old girl who developed hypoxemia during OLV, which was not corrected by the usual maneuvers. Hypoxemia was successfully treated with a phenylephrine infusion. The potential applications of $\alpha$-adrenergic agonists such as phenylephrine in the treatment of hypoxemia during OLV are discussed and its physiologic basis reviewed.
\end{abstract}

\section{Introduction}

One-lung ventilation (OLV) is frequently used during thoracic surgery to facilitate surgical exposure. The ventilationperfusion mismatch that results from perfusion of the nonventilated lung can cause significant transpulmonary shunting and hypoxemia. Significant hypoxemia, defined as an oxygen saturation less than $90 \%$, occurs in 5 to $10 \%$ of patients during OLV. ${ }^{1,2}$ The effective management of hypoxemia during OLV remains a challenge for the anesthesiologist, but an understanding of the various oxygenation strategies and their implementation in a systematic fashion helps to avoid the need to abandon OLV. We present a 12-year-old girl who developed severe hypoxemia during OLV that was not corrected by the usual maneuvers including oxygen insufflations into the surgical lung. Hypoxemia was successfully treated with a phenylephrine infusion. The potential applications of $\alpha$-adrenergic agonists such as phenylephrine in the treatment of hypoxemia during OVL are discussed and its physiologic basis reviewed.

\section{Case Report}

Institutional Review Board approval for publication of single case reports is not required at Nationwide Children's Hospital. An otherwise healthy 12-year-old, $35 \mathrm{~kg}$, girl was scheduled for anterior-posterior spinal fusion. Preoperative examination revealed idiopathic scoliosis with no history of cardiopulmonary disease. A thoracoscopic approach was planned for the anterior portion of the patient. Therefore, following received

December 18, 2012

accepted after revision

February 18, 2013

published online

April 15, 2013
DOI http://dx.doi.org/

10.1055/s-0033-1343734. ISSN 2194-7635. (c) 2013 Georg Thieme Verlag KG Stuttgart · New York
License terms

(®) $\Theta \circledast$ 
anesthetic induction, her trachea was intubated with a 32 French, double-lumen endotracheal tube (DLT), the position of which was confirmed using bronchoscopic visualization in addition to standard auscultation. Correct positioning was further confirmed with fiberoptic bronchoscopy after the patient was positioned in the left lateral decubitus position. Maintenance anesthesia was achieved with infusions of propofol, dexmedetomidine, and remifentanil. Pressure control ventilation was initiated with a peak inspiratory pressure of $12 \mathrm{~cm} \mathrm{H}_{2} \mathrm{O}$, tidal volume of $8 \mathrm{~mL} / \mathrm{kg}$, fraction of inspired oxygen of 1.0 , respiratory rate of 12 breaths per minute, and positive end expiratory pressure (PEEP) of $4 \mathrm{~cm} \mathrm{H}_{2} \mathrm{O}$. OLV was initiated and the inspiratory pressure was adjusted to $14 \mathrm{~cm}$ $\mathrm{H}_{2} \mathrm{O}$ to maintain a tidal volume of $8 \mathrm{~mL} / \mathrm{kg}$. The patient's oxygen saturation remained at $100 \%$ for approximately 10 minutes after the initiation of OLV. During the following 3 minutes, and before surgical incision, the patient's oxygen saturation rapidly decreased from 100 to 64\%. Two-lung ventilation was immediately resumed with a prompt return of the oxygen saturation to $100 \%$. Mean arterial pressure (MAP) remained stable throughout this time at 55 to $65 \mathrm{~mm}$ $\mathrm{Hg}$. Fiber optic bronchoscopy confirmed correct positioning of the DLT. OLV was then resumed with an increase of the PEEP to $7 \mathrm{~cm} \mathrm{H}_{2} \mathrm{O}$ and peak inspiratory pressure of 14 to $15 \mathrm{~cm}$ $\mathrm{H}_{2} \mathrm{O}$ to achieve tidal volumes of 10 to $11 \mathrm{~mL} / \mathrm{kg}$. With these settings, the patient's oxygen saturation slowly decreased to a low of $88 \%$ during the next 10 minutes. Continuous positive airway pressure (CPAP) was then applied to the nondependent lung, which increased the oxygen saturation to 90 to $92 \%$. The surgeon was allowed to begin video-assisted thoracoscopy and the patient's oxygenation remained stable. Approximately 10 minutes after the incision, the surgeon noted that the nondependent lung was overinflated, preventing surgical access to the anterior portion of the spine. CPAP was discontinued and surgical visualization improved. However, the oxygen saturations decreased to 85 to $88 \%$. Hemodynamic function remained stable with a MAP of $60 \mathrm{~mm} \mathrm{Hg}$. A $50-\mu \mathrm{g}$ bolus of phenylephrine was administered in an attempt to improve oxygen saturation and avoid the need to abort OLV. The MAP increased to 70 to $75 \mathrm{~mm} \mathrm{Hg}$ with an immediate improvement in oxygen saturation to $94 \%$. A phenylephrine infusion was started at $0.1 \mu \mathrm{g} / \mathrm{kg} / \mathrm{min}$ and titrated to $0.3 \mu \mathrm{g} / \mathrm{kg} / \mathrm{min}$ with the oxygen saturation reaching a high of $96 \%$ with an MAP of $80 \mathrm{~mm} \mathrm{Hg}$. The phenylephrine was continued at $0.3 \mu \mathrm{g} / \mathrm{kg} / \mathrm{min}$ for the remainder of the anterior portion of the surgical procedure, which lasted 2 to 3 hours. During this time, the oxygen saturations were stable at 96 to $98 \%$. At the conclusion of the anterior component of the procedure, two-lung ventilation was resumed, the phenylephrine infusion was discontinued, and the DLT was exchanged for a standard single-lumen endotracheal tube. The posterior portion of the surgical procedure was completed uneventfully. The patient's trachea was extubated in the operating room and she was transported to the postanesthesia care unit (PACU). In the PACU, her oxygen saturation remained at $100 \%$ on $1 \mathrm{~L} / \mathrm{min}$ oxygen administered via nasal cannula. She was discharged to the inpatient ward in a stable condition and the remainder of postoperative course was unremarkable.

\section{Discussion}

With advances in thoracic surgical techniques, certain requirements have been placed on anesthetic care. During thoracoscopy, effective OLV is generally required to allow for adequate surgical visualization. Therefore, a systematic approach to the treatment of hypoxemia is needed. As was illustrated in our case, severe hypoxemia during OLV should be immediately treated by the administration of $100 \%$ oxygen and restoration of two-lung ventilation. Examination with fiberoptic bronchoscopy was performed to rule out DLT malposition and mechanical obstruction. Additional attempts of OLV should be combined with strategies to improve ventilation-perfusion mismatching including elimination of dependent lung atelectasis with PEEP and recruitment maneuvers, ${ }^{3,4}$ application of CPAP or low flow oxygen insufflations to the nondependent (surgical) lung, ${ }^{5}$ elimination of volatile anesthetic agents, ${ }^{6,7}$ and in specific circumstances the addition of inhaled nitric oxide (iNO) to the intraoperative gas regimen. ${ }^{8,9}$

As demonstrated by our case, these methods are not universally effective nor are they without drawbacks. Increasing PEEP to the dependent lung has the potential to paradoxically worsen hypoxemia by increasing shunt fraction through the nondependent lung via its effects on pulmonary vascular resistance. ${ }^{10}$ Recruitment maneuvers may have the same effect as well as cause transient decreases in cardiac output. ${ }^{3,4}$ Application of CPAP or low flow oxygen insufflations to the nondependent lung can often create undesirable surgical conditions, especially during video-assisted thoracoscopy. Elimination of volatile anesthetic agents and converting to a total intravenous anesthetic has the theoretical advantage of improving hypoxic pulmonary vasoconstriction (HPV), though this is controversial ${ }^{11,12}$ and unsupported by a 2008 Cochrane Database review. ${ }^{13}$ The intraoperative use of iNO has the potential advantage of reducing shunt fraction by selectively vasodilating only the areas of the lung that are perfused with negligible effects on the systemic circulation. ${ }^{14}$ However, issues include cost and the logistics of the additional equipment that is required for its administration, which may not be readily available in all operating rooms. ${ }^{15}$

In our case, recruitment of alveoli in the dependent lung via increased tidal volumes and the addition of PEEP was unsuccessful in increasing the oxygen saturation. Although low flow oxygen insufflation to the operative lung was not successful, the oxygen saturation increased with application of CPAP to the nondependent lung. However, interference with surgical exposure necessitated the discontinuation of CPAP. With discontinuation of CPAP to the nondependent lung, recruitment maneuvers alone proved to be inadequate in maintaining adequate oxygen saturation. With the CPAP off, the patient's saturation was not low enough to require immediate return to two-lung ventilation, although the slow trend downward to less than 90\% suggested that another oxygenation strategy was necessary.

Almitrine is a peripheral chemoreceptor agonist that has been shown to increase pulmonary vascular resistance without significant systemic hemodynamic consequence. ${ }^{16}$ 
Human studies have demonstrated almitrine's ability to augment HPV and improve oxygenation during adult respiratory distress syndrome (ARDS) and OLV, both alone and in combination with iNO. ${ }^{14,17,18}$ Unfortunately, almitrine has also been associated with peripheral neuropathy after prolonged use and is not available in the United States. Although almitrine is not available to us for clinical use, the concept of pharmacologic augmentation of HPV remains an interesting possibility. It has been suggested that even a nonselective vasoconstrictor, when given alone, could improve oxygenation by augmenting HPV. ${ }^{19,20}$

Phenylephrine is an $\alpha$-adrenergic receptor agonist with nonselective vasoconstricting properties on both the pulmonary and systemic circulation. To our knowledge, only one group of investigators has prospectively studied phenylephrine as a therapy for augmenting HPV in hypoxemic patients. ${ }^{21}$ Doering et al evaluated the effects of phenylephrine, iNO, and the combination of the two in the treatment of hypoxemia in adults with ARDS. Twelve patients with ARDS were given phenylephrine, iNO, and the combination of the two in random order. Although all therapies improved the $\mathrm{PaO}_{2}, 6$ of the 12 patients were classified as "phenylephrine responders." Among the phenylephrine responders, the $\mathrm{PaO}_{2}$ increased by an average of $27 \mathrm{~mm} \mathrm{Hg}$ ( $p<0.05$ when compared with baseline). Inhaled $\mathrm{NO}$ increased $\mathrm{PaO}_{2}$ by an average of $33 \mathrm{~mm} \mathrm{Hg}$ ( $p=$ nonsignificant when compared with phenylephrine), whereas the combination of the two agents increased $\mathrm{PaO}_{2}$ by an average of $55 \mathrm{~mm} \mathrm{Hg}(p<0.05$ compared with either agent alone).

In a similar case report, Sato and Kato ${ }^{22}$ presented a 59-year-old woman who was hypoxic while undergoing clipping of a cerebral aneurysm. A phenylephrine infusion was used, which increased $\mathrm{PaO}_{2}$ from 100 to $150 \mathrm{~mm} \mathrm{Hg}$. Interestingly, they reported that the $\mathrm{PaO}_{2}$ decreased back to $100 \mathrm{~mm} \mathrm{Hg}$ when the phenylephrine infusion was discontinued at the end of the surgery.

The effect of phenylephrine may represent the selective enhancement of HPV and may be beneficial in improving oxygenation when ventilation-perfusion inequalities are present related either to pulmonary parenchymal disease (ARDS) or OLV. Given the difficulties faced by anesthesiologists during OLV, pharmacologic augmentation of HPV could prove to be of great clinical benefit. Although anecdotal, our experience demonstrates that phenylephrine should be considered to improve oxygenation during OLV when conventional techniques fail. In our case, the addition of phenylephrine allowed for the use of OLV and successful completion of the thoracoscopic portion of the procedure. We suggest that the use of phenylephrine to enhance HPV and maintain oxygenation during OLV warrants further prospective investigation.

\section{References}

1 Karzai W, Schwarzkopf K. Hypoxemia during one-lung ventilation: prediction, prevention, and treatment. Anesthesiology 2009;110 (6):1402-1411
2 Slinger P, Suissa S, Triolet W. Predicting arterial oxygenation during one-lung anaesthesia. Can J Anaesth 1992;39(10):1030-1035

3 Tusman G, Böhm SH, Sipmann FS, Maisch S. Lung recruitment improves the efficiency of ventilation and gas exchange during onelung ventilation anesthesia. Anesth Analg 2004;98(6):1604-1609

4 Cinnella G, Grasso S, Natale C, et al. Physiological effects of a lungrecruiting strategy applied during one-lung ventilation. Acta Anaesthesiol Scand 2008;52(6):766-775

5 Hogue CW Jr. Effectiveness of low levels of nonventilated lung continuous positive airway pressure in improving arterial oxygenation during one-lung ventilation. Anesth Analg 1994;79 (2):364-367

6 Domino KB, Borowec L, Alexander CM, et al. Influence of isoflurane on hypoxic pulmonary vasoconstriction in dogs. Anesthesiology 1986;64(4):423-429

7 Kellow NH, Scott AD, White SA, Feneck RO. Comparison of the effects of propofol and isoflurane anaesthesia on right ventricular function and shunt fraction during thoracic surgery. $\mathrm{Br} \mathrm{J}$ Anaesth 1995;75(5):578-582

8 Ichinose F, Roberts JD Jr, Zapol WM. Inhaled nitric oxide: a selective pulmonary vasodilator: current uses and therapeutic potential. Circulation 2004;109(25):3106-3111

9 Dietrich CC, Tobias JD. Intraoperative administration of nitric oxide. J Intensive Care Med 2003;18(3):146-149

10 Inomata S, Nishikawa T, Saito S, Kihara S. "Best" PEEP during onelung ventilation. Br J Anaesth 1997;78(6):754-756

11 Schwarzkopf K, Hueter L, Schreiber T, Preussler NP, Loeb V, Karzai W. Oxygenation during one-lung ventilation with propofol or sevoflurane. Middle East J Anaesthesiol 2009;20(3):397-400

12 Pruszkowski O, Dalibon N, Moutafis M, et al. Effects of propofol vs sevoflurane on arterial oxygenation during one-lung ventilation. Br J Anaesth 2007;98(4):539-544

13 Bassi A, Milani WR, El Dib R, Matos D. Intravenous versus inhalation anaesthesia for one-lung ventilation. Cochrane Database Syst Rev 2008;(2):CD00631310.1002/14651858.CD006313

14 Moutafis M, Liu N, Dalibon N, et al. The effects of inhaled nitric oxide and its combination with intravenous almitrine on Pao2 during one-lung ventilation in patients undergoing thoracoscopic procedures. Anesth Analg 1997;85(5):1130-1135

15 Tobias JD, Grueber R. Nitric oxide administration using an anesthesia machine ventilator. Am J Anesthesiol 2000;27:137-139

16 Gluskowski J, Gorecka D, Hawrylkiewicz I, Zielinski J. Acute effects of almitrine infusion on pulmonary haemodynamics in normal man. Bull Eur Physiopathol Respir 1984;20(4):313-317

17 Dalibon N, Moutafis M, Liu N, Law-Koune JD, Monsel S, Fischler M. Treatment of hypoxemia during one-lung ventilation with almitrine. Anesth Analg 2004;98(3):590-594

18 Roch A, Papazian L, Bregeon F, et al. High or low doses of almitrine bismesylate in ARDS patients responding to inhaled NO and receiving norepinephrine? Intensive Care Med 2001;27(11): 1737-1743

19 Marshall BE, Hanson CW, Frasch F, et al. Role of hypoxic pulmonary vasoconstriction in pulmonary gas exchange and blood flow distribution. 2. Pathophysiology. Intensive Care Med 1994;20(5): 379-389

20 Marshall BE, Chen L, Frasch HF, et al. Pulmonary vasoconstriction enhances the response to nitric oxide in atelectasis. (abstract) Anesthesiology 1995;83:A1196

21 Doering EB, Hanson CW III, Reily DJ, Marshall C, Marshall BE. Improvement in oxygenation by phenylephrine and nitric oxide in patients with adult respiratory distress syndrome. Anesthesiology 1997;87(1):18-25

22 Sato K, Kato M. [Improvement in oxygenation by alpha stimulant]. Masui 2001;50(10):1118-1120 (in Japanese) 